\title{
High-Dimensional D. H. Lehmer Problem over Quarter Intervals
}

\author{
Tianping Zhang \\ College of Mathematics and Information Science, Shaanxi Normal University, Xian, Shaanxi 710119, China
}

Correspondence should be addressed to Tianping Zhang; tpzhang@snnu.edu.cn

Received 9 January 2014; Accepted 31 March 2014; Published 24 April 2014

Academic Editor: Soon-Yeong Chung

Copyright (C) 2014 Tianping Zhang. This is an open access article distributed under the Creative Commons Attribution License, which permits unrestricted use, distribution, and reproduction in any medium, provided the original work is properly cited.

The high-dimensional D. H. Lehmer problem over quarter intervals is studied. By using the properties of character sum and the estimates of Dirichlet $L$-function, the previous result is improved to be the best possible in the case of $q=p$, an odd prime with $p \equiv 1(\bmod 4)$, which is shown by studying the mean square value of the error term.

\section{Introduction and Main Results}

Let $q>2$ be an odd integer and let $a$ be an integer coprime to $q$. For each integer $b$ with $1 \leq b<q$ and $(b, q)=1$, there is a unique integer $c$ with $1 \leq c<q$ such that $b c \equiv$ $a(\bmod q)$. Let $M(a, q)$ denote the number of solutions $(b, c)$ of the congruence equation $b c \equiv a(\bmod q)$ with $1 \leq b, c<q$ such that $b, c$ are of opposite parity. D. H. Lehmer posed the problem to find $M(1, p)$ or at least to say something nontrivial about it (see problem F12 of [1], page 251), where $p$ is an odd prime. Zhang [2] proved that

$$
M(1, q)=\frac{\phi(q)}{2}+O\left(q^{1 / 2} d^{2}(q) \ln ^{2} q\right),
$$

where $\phi(q)$ is the Euler function and $d(q)$ is the Dirichlet divisor function. For the further properties of $M(a, p)$, he [3] studied the mean square value of the error term $M(a, p)-(p-$ 1)/2 and obtained

$$
\sum_{a=1}^{p-1}\left(M(a, p)-\frac{p-1}{2}\right)^{2}=\frac{3}{4} p^{2}+O\left(p \exp \left(\frac{3 \ln p}{\ln \ln p}\right)\right) .
$$

For general odd integer $q$, the similar properties of $M(a, q)$ were studied in [4].

It is interesting to study the $\mathrm{D}$. H. Lehmer problem over short intervals $[1, \lambda q]$ with $0<\lambda \leq 1$ being a real number. Denote by $N(a, q)$ the number of pairs of integers $b, c$ with $b c \equiv a(\bmod q), 1 \leq b, c \leq(q-1) / 2$, and $b, c$ having different parity. In [5], Xu and Zhang studied the mean square value of error term

$$
E(a, q)=N(a, q)-\frac{1}{8} \phi(q)
$$

in the case of $q=p$ and obtained a sharp asymptotic formula

$$
\sum_{a=1}^{p-1} E^{2}(a, p)=\frac{9}{64} p^{2}+O\left(p^{1+\epsilon}\right) .
$$

Let $k$ be a positive integer and $n$ a nonnegative integer; let $0<\lambda_{1}, \ldots, \lambda_{k+1} \leq 1$ be real numbers and $\mathbf{w}=$ $\left(\lambda_{1}, \lambda_{2}, \ldots, \lambda_{k+1}\right)$. Let $q \geq \max \left\{\left[1 / \lambda_{i}\right]: 1 \leq i \leq k+1\right\}$ be a positive integer and $a$ an integer coprime to $q$. In [6], Xu and Zhang studied the high-dimensional D. H. Lehmer problem over short intervals as

$$
N(a, k, \mathbf{w}, q, n)=\sum_{b_{1}=1}^{\left[\lambda_{1} q\right]} \cdots \sum_{\substack{\left.b_{k}=1 \\ b_{1} \cdots b_{k} c \equiv a \bmod q\right) \\ 2 \nmid\left(b_{1}+\cdots+b_{k}+c\right)}}^{\left[\lambda_{k} q\right]\left[\lambda_{k=1}, \lambda_{k+1} q\right]}\left(b_{1} \cdots b_{k}-c\right)^{2 n},
$$

and obtained an interesting asymptotic formula

$$
\begin{aligned}
N & (a, k, \mathbf{w}, q, n) \\
& =C(k, \mathbf{w}, n) \phi^{k}(q) q^{2 k n}+O\left(4^{n} q^{(2 n+1) k-1 / 2} d^{2}(q) \ln q\right),
\end{aligned}
$$


where

$$
C(k, \mathbf{w}, n)= \begin{cases}\frac{\left(\lambda_{1} \cdots \lambda_{k}\right)^{2 n+1} \lambda_{k+1}}{2(2 n+1)^{k}}, & \text { if } k \geq 2 ; \\ \frac{\lambda_{1}^{2 n+2}+\lambda_{2}^{2 n+2}-\left(\lambda_{1}-\lambda_{2}\right)^{2 n+2}}{4(n+1)(2 n+1)}, & \text { if } k=1 .\end{cases}
$$

They also improved the result for $N_{1 / 2}(a, k, q)=N(a, k$, $\mathbf{w}, q, 0)$ in the case $\mathbf{w}=(1 / 2,1 / 2, \ldots, 1 / 2)$ to be the best possible, by studying the mean square value of the error term $E_{1 / 2}(a, k, q)=N_{1 / 2}(a, k, q)-\phi^{k}(q) / 2^{k+2}$.

In this paper, we consider the high-dimensional D. $\mathrm{H}$. Lehmer problem over quarter intervals. Let $N_{1 / 4}(a, k, q)=$ $N(a, k, \mathbf{w}, q, 0)$ in the case $\mathbf{w}=(1 / 4,1 / 4, \ldots, 1 / 4)$. That is,

$$
N_{1 / 4}(a, k, q)=\sum_{b_{1}=1} \sum_{\substack{b_{2}=1 \\ b_{1} b_{2} \cdots b_{k} c \equiv a(\bmod q) \\ 2 \nmid\left(b_{1}+b_{2}+\cdots+b_{k}+c\right)}}^{(q-1) / 4} \sum_{\substack{(q-1) / 4 \\(q-1) / 4}}^{(q-1) / 4} 1 .
$$

We will use the properties of character sum and the estimates of Dirichlet $L$-function to obtain a sharper asymptotic formula of $N_{1 / 4}(a, k, q)$ in the case of $q=p$, an odd prime with $p \equiv 1(\bmod 4)$. In order to show that our result is close to be the best possible, the mean square value of $N_{1 / 4}(a, k, p)-$ $\phi^{k}(p) / 2^{2 k+3}$ is studied too.

In this paper, we will use the following notations:

$\sum_{\chi \bmod q, \chi(-1)=-1}^{*}$ denotes the summation over all primitive characters modulo $q$ such that $\chi(-1)=-1$;

$J(q)$ denotes the number of all primitive characters modulo $q$;

$d_{k}(n)$ denotes the $k$-th divisor function (i.e., the number of solutions of the equation $n_{1} n_{2} \cdots n_{k}=n$ in positive integers $n_{1}, n_{2}, \ldots, n_{k}$ );

$\chi_{4}$ denotes the primitive character modulo 4 ;

$\chi_{8_{1}}$ denotes the primitive character modulo 8 with $\chi_{8_{1}}(-1)=-1$, and $\chi_{8_{2}}$ denotes the primitive character modulo 8 with $\chi_{8_{2}}(-1)=1$.

The main results are the following.

Theorem 1. Let $p \geq 5$ be an odd prime with $p \equiv 1(\bmod 4)$ and a coprime to $p$. Then, for any positive integer $k$ with $(p, k(k+1))=1$, one has the asymptotic formula that

$$
N_{1 / 4}(a, k, p)=\frac{p^{k}}{2^{2 k+3}}+O\left(2^{k^{2}+2 k+2} k p^{k / 2} \ln ^{k+1} p\right) .
$$

Theorem 2. Let $p \geq 5$ be an odd prime with $p \equiv 1(\bmod 4)$. Then, for any positive integer $k$, one has

$$
\sum_{a=1}^{p-1}\left|E_{1 / 4}(a, k, p)\right|^{2}=A(k) p^{k+1}+O_{k}\left(p^{k+\epsilon}\right),
$$

where

$$
\begin{aligned}
A(k)= & \left(\frac{1}{8}-2^{k-1}+(-1)^{k} 2^{k-1}\right) \sum_{\substack{n=1 \\
(n, 2 p)=1}}^{\infty} \frac{d_{k+1}^{2}(n)}{n^{2}} \\
& +\sum_{i=0}^{k+1} C_{k+1}^{i} \sum_{j=0}^{i} 6^{j} \sum_{s=0}^{i-j} \sum_{t=0}^{k+1-i} C_{i-j}^{s} C_{k+1-i}^{t} \\
& \times \frac{(-1)^{k+1-i}}{2^{|2 s+4 t+i+j-2 k-2|+k+i+5}} \\
& \times \sum_{\substack{n=1 \\
(n, p)=1}}^{\infty} \frac{d_{k+1}\left(2^{|2 s+4 t+i+j-2 k-2|} n\right) d_{k+1}(n)}{n^{2}}
\end{aligned}
$$$$
+\sum_{j_{1}=0}^{k+1} \sum_{l_{1}=0}^{j_{1}} \sum_{l_{2}=0}^{k+1} C_{k+1}^{j_{1}} C_{j_{1}}^{l_{1}} C_{k+1}^{l_{2}} \frac{(-1)^{k+j_{1}+l_{2}}}{2^{\left|2 k+2+l_{2}-j_{1}-l_{1}\right|+k+3-l_{1}-l_{2}}}
$$$$
\times \sum_{n=1}^{\infty} \frac{d_{k+1}\left(2^{\left|2 k+2+l_{2}-j_{1}-l_{1}\right|} n\right) d_{k+1}(n)}{n^{2}}
$$$$
(n, p)=1
$$$$
+\sum_{j_{1}=0}^{k+1} \sum_{j_{2}=1}^{k} \sum_{l_{1}=0}^{j_{1}} \sum_{l_{2}=0}^{j_{2}} C_{k+1}^{j_{1}} C_{k+1}^{j_{2}} C_{j_{1}}^{l_{1}} C_{j_{2}}^{l_{2}}
$$$$
\times(-1)^{k+j_{1}+l_{2}} \sqrt{2}^{2 j_{1}+j_{2}+4 l_{1}-7 k-11}
$$$$
\times \sum_{\substack{n=1 \\(n, p)=1}}^{\infty} \frac{r_{j_{2}}\left(2^{3 k+3+l_{2}-j_{1}-j_{2}-l_{1}} n\right) d_{k+1}(n)}{n^{2}}
$$$$
+\sum_{j_{2}=1}^{k+1}(-1)^{k+j_{2}} \sqrt{2}^{2 k+j_{2}+2} C_{k+1}^{j_{2}} \sum_{\substack{n=1 \\(n, p)=1}}^{\infty} \frac{d_{k+1}(n) r_{j_{2}}(n)}{n^{2}}
$$$$
+\sum_{l_{1}=0}^{k+1} \sum_{l_{2}=0}^{k+1} C_{k+1}^{l_{1}} C_{k+1}^{l_{2}} \frac{(-1)^{k+l_{1}+l_{2}}}{2^{\left|l_{1}-l_{2}\right|+2-l_{1}-l_{2}}}
$$$$
\times \sum_{\substack{n=1 \\(n, p)=1}}^{\infty} \frac{d_{k+1}\left(2^{\left|l_{1}-l_{2}\right|} n\right) d_{k+1}(n)}{n^{2}}
$$$$
+\sum_{\substack{j_{2}=0 \\ j_{2}-l_{2} \geq k+1}}^{k} \sum_{l_{1}=0}^{k+1} \sum_{l_{2}=0}^{j_{2}}(-1)^{k+l_{1}+l_{2}} C_{k+1}^{j_{2}} C_{k+1}^{l_{1}} C_{j_{2}}^{l_{2}} \sqrt{2}^{3 k+4 l_{2}+1-3 j_{2}}
$$

$$
\begin{aligned}
& \times \sum_{\substack{n=1 \\
(n, p)=1}}^{\infty} \frac{d_{k+1}\left(2^{\left(j_{2}-l_{2}\right)-\left(k+1-l_{1}\right)} n\right) r_{j_{2}}(n)}{n^{2}} \\
& +\sum_{\substack{j_{2}=0 \\
j_{2}-l_{2}<k+1-l_{1}}}^{k} \sum_{l_{1}=0}^{k+1} \sum_{l_{2}=0}^{j_{2}}(-1)^{k+l_{1}+l_{2}} C_{k+1}^{j_{2}} C_{k+1}^{l_{1}} C_{j_{2}}^{l_{2}} \sqrt{2}^{4 l_{1}+j_{2}-k-3} \\
&
\end{aligned}
$$




$$
\begin{aligned}
& \times \sum_{\substack{n=1 \\
(n, p)=1}}^{\infty} \frac{d_{k+1}(n) r_{j_{2}}\left(2^{\left(k+1-l_{1}\right)-\left(j_{2}-l_{2}\right)} n\right)}{n^{2}}
\end{aligned}
$$

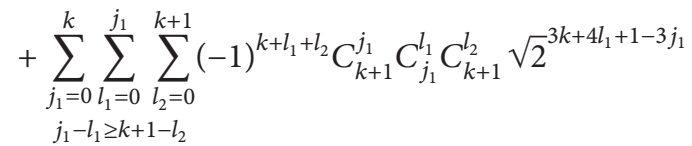

$$
\begin{aligned}
& \times \sum_{\substack{n=1 \\
(n, p)=1}}^{\infty} \frac{d_{k+1}\left(2^{\left(j_{1}-l_{1}\right)-\left(k+1-l_{2}\right)} n\right) r_{j_{2}}(n)}{n^{2}} \\
& +\sum_{\substack{j_{1}=0 \\
j_{1}-l_{1}<k+1-l_{2}}}^{k} \sum_{l_{1}=0}^{j_{1}} \sum_{l_{2}=0}^{k+1}(-1)^{k+l_{1}+l_{2}} C_{k+1}^{j_{1}} C_{j_{1}}^{l_{1}} C_{k+1}^{l_{2}} \sqrt{2}^{4 l_{2}+j_{1}-k-3} \\
& \times \sum_{\substack{n=1 \\
(n, p)=1}}^{\infty} \frac{d_{k+1}(n) r_{j_{2}}\left(2^{\left(k+1-l_{2}\right)-\left(j_{1}-l_{1}\right)} n\right)}{n^{2}} \\
& +\sum_{j_{1}=1}^{k} \sum_{\substack{j_{2}=1 \\
j_{1}-l_{1} \geq j_{2}-l_{2}}}^{k} \sum_{l_{1}=0}^{j_{1}} \sum_{l_{2}=0}^{j_{2}}(-1)^{k+l_{1}+l_{2}} \\
& \times C_{k+1}^{j_{1}} C_{k+1}^{j_{2}} C_{j_{1}}^{l_{1}} C_{j_{2}}^{l_{2}} \sqrt{2}^{2 k+4 l_{1}+j_{2}-3 j_{1}} \\
& \times \sum_{\substack{n=1 \\
(n, p)=1}}^{\infty} \frac{r_{j_{1}-l_{1}}(n) r_{j_{2}-l_{2}}\left(2^{\left(j_{1}-l_{1}\right)-\left(j_{2}-l_{2}\right)} n\right)}{n^{2}} \\
& +\sum_{j_{1}=1}^{k} \sum_{\substack{j_{2}=1 \\
j_{1}-l_{1}<j_{2}-l_{2}}}^{k} \sum_{l_{1}=0}^{j_{1}} \sum_{l_{2}=0}^{j_{2}}(-1)^{k+l_{1}+l_{2}} \\
& \times C_{k+1}^{j_{1}} C_{k+1}^{j_{2}} C_{j_{1}}^{l_{1}} C_{j_{2}}^{l_{2}} \sqrt{2}^{2 k+4 l_{1}+j_{2}-3 j_{1}} \\
& \times \sum_{\substack{n=1 \\
(n, p)=1}}^{\infty} \frac{r_{j_{2}-l_{2}}(n) r_{j_{1}-l_{1}}\left(2^{\left(j_{2}-l_{2}\right)-\left(j_{1}-l_{1}\right)} n\right)}{n^{2}}
\end{aligned}
$$

and where $C_{k}^{j}=k ! / j !(k-j) !, r_{j}(n)=\sum_{t \mid n} d_{j}(t) d_{k-j}(n / t)$ $\chi_{8_{2}}(n / t)$, and $\epsilon$ is any fixed positive number.

From Theorem 2 we know that $\left|E_{1 / 4}(a, k, p)\right| \gg p^{k / 2}$ for some $a$ and thus the bound in Theorem 1 is close to be the best possible.

For general odd number $q \geq 3$, whether there exist similar asymptotic formulae for $N(a, k, \mathbf{w}, q, 0)$ in the cases of $\mathbf{w}=(1 / 2,1 / 2, \ldots, 1 / 2)$ and $\mathbf{w}=(1 / 4,1 / 4, \ldots, 1 / 4)$ are open problems.

\section{Several Auxiliary Lemmas}

To establish the main results of our theorems, we need the following several auxiliary lemmas.

Lemma 3. Let $q \geq 9$ be an odd integer and let $\chi$ be a primitive Dirichlet character modulo q. Then one has

$$
\begin{aligned}
& \sum_{b=1}^{[q / 4]} \chi(b)=\left\{\begin{array}{lr}
\frac{\tau(\chi)}{\pi} L\left(1, \bar{\chi} \chi_{4}\right) & \text { if } \chi(-1)=1 ; \\
2 i \pi & (1, \bar{\chi}) \\
\times(2+\bar{\chi}(2)-\bar{\chi}(4)) & \text { if } \chi(-1)=-1,
\end{array}\right. \\
& \sum_{b=1}^{[q / 8]} \chi(b) \\
& =\left\{\begin{array}{rr}
\frac{\tau(\chi)}{2 \pi}\left(\bar{\chi}(2) L\left(1, \bar{\chi} \chi_{4}\right)+\sqrt{2} L\left(1, \bar{\chi} \chi_{8_{1}}\right)\right) \\
\begin{array}{rr}
\frac{\tau(\chi)}{4 i \pi}\left((4+\bar{\chi}(4)-\bar{\chi}(8)) L(1, \bar{\chi})-2 \sqrt{2} L\left(1, \bar{\chi} \chi_{8_{2}}\right)\right)
\end{array} \\
\text { if } \chi(-1)=-1 .
\end{array}\right.
\end{aligned}
$$

Proof. See [7] or Lemma 2 in [8].

Lemma 4. Let $p \geq 5$ be an odd prime with $p \equiv 1(\bmod 4)$ and a coprime to $p$. Then, for any positive integer $k$, one has

$$
\begin{aligned}
& E_{1 / 4}(a, k, p) \\
& =\frac{1}{2(2 i \pi)^{k+1} \phi(p)} \\
& \times \sum_{\substack{\chi \bmod p \\
\chi(-1)=-1}} \bar{\chi}(a)(2+\bar{\chi}(2)-\bar{\chi}(4))^{k+1} \tau^{k+1}(\chi) L^{k+1}(1, \bar{\chi}) \\
& +\frac{1}{2 \pi^{k+1} \phi(p)} \sum_{\substack{\chi \bmod p \\
\chi(-1)=1 \\
\chi \neq \chi_{0}}} \bar{\chi}(a) \tau^{k+1}(\chi) L^{k+1}\left(1, \bar{\chi} \chi_{4}\right) \\
& +O\left(\frac{1}{p}\right)-\frac{\sqrt{2}^{k-1}}{\pi^{k+1} \phi(p)} \\
& \times \sum_{\substack{\chi \bmod p \\
\chi(-1)=1 \\
\chi \neq \chi_{0}}} \bar{\chi}(a) \chi^{k+1}(2) \tau^{k+1}(\chi) L^{k+1}\left(1, \bar{\chi} \chi_{8_{1}}\right)
\end{aligned}
$$




$$
\begin{aligned}
& -\frac{1}{2(i \pi)^{k+1} \phi(p)} \\
& \times \sum_{\substack{\chi \bmod p \\
\chi(-1)=-1}} \bar{\chi}(a) \tau^{k+1}(\chi) \\
& \times\left((2 \chi(2)-1) L(1, \bar{\chi})-\sqrt{2} \chi(2) L\left(1, \bar{\chi} \chi_{8_{2}}\right)\right)^{k+1} .
\end{aligned}
$$

Proof. From the definition of $N_{1 / 4}(a, k, p)$ we have

$$
\begin{aligned}
& N_{1 / 4}(a, k, p) \\
& =\frac{1}{2} \sum_{b_{1}=1}^{(p-1) / 4} \sum_{\substack{b_{2}=1 \\
b_{1} b_{2} \cdots b_{k} c \equiv a(\bmod p)}}^{(p-1) / 4} \sum_{b_{k}=1}^{(p-1) / 4} \sum_{c=1}^{(p-1) / 4}\left(1-(-1)^{b_{1}+b_{2}+\cdots+b_{k}+c}\right) \\
& =\frac{1}{2 \phi(p)}\left(\sum_{\chi \bmod p} \bar{\chi}(a)\left(\sum_{b=1}^{(p-1) / 4} \chi(b)\right)^{k+1}\right. \\
& \left.-\sum_{\chi \bmod p} \bar{\chi}(a)\left(\sum_{b=1}^{(p-1) / 4}(-1)^{b} \chi(b)\right)^{k+1}\right) \\
& =\frac{\phi^{k}(p)}{2^{2 k+3}}+\frac{1}{2 \phi(p)} \sum_{\substack{\chi \bmod p \\
\chi \neq \chi_{0}}} \bar{\chi}(a)\left(\sum_{b=1}^{(p-1) / 4} \chi(b)\right)^{k+1} \\
& -\frac{1}{2 \phi(p)} \sum_{\chi \bmod p} \bar{\chi}(a)\left(\sum_{b=1}^{(p-1) / 4}(-1)^{b} \chi(b)\right)^{k+1} .
\end{aligned}
$$

Note that $p \equiv 1(\bmod 4)$; we have

$$
\begin{aligned}
& \sum_{b=1}^{(p-1) / 4}(-1)^{b} \chi(b) \\
& =2 \sum_{b=1,2 \mid b}^{(p-1) / 4} \chi(b)-\sum_{b=1}^{(p-1) / 4} \chi(b) \\
& =2 \chi(2) \sum_{b=1,2 \mid b}^{(p-1) / 8} \chi(b)-\sum_{b=1}^{(p-1) / 4} \chi(b)
\end{aligned}
$$

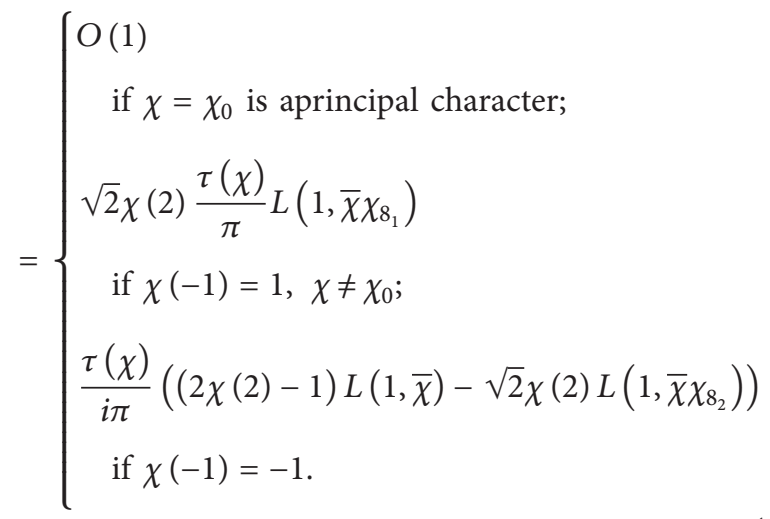

Combining the above with Lemma 3, we can get

$$
\begin{aligned}
& N(a, k, p) \\
& =\frac{\phi^{k}(p)}{2^{2 k+3}}+\frac{1}{2(2 i \pi)^{k+1} \phi(p)} \\
& \times \sum_{\substack{\chi \bmod p \\
\chi(-1)=-1}} \bar{\chi}(a)(2+\bar{\chi}(2)-\bar{\chi}(4))^{k+1} \tau^{k+1}(\chi) L^{k+1}(1, \bar{\chi}) \\
& +\frac{1}{2 \pi^{k+1} \phi(p)} \\
& \times \sum_{\substack{\chi \bmod p \\
\chi(-1)=1 \\
\chi \neq \chi_{0}}} \bar{\chi}(a) \tau^{k+1}(\chi) L^{k+1}\left(1, \bar{\chi} \chi_{4}\right)+O\left(\frac{1}{p}\right)
\end{aligned}
$$

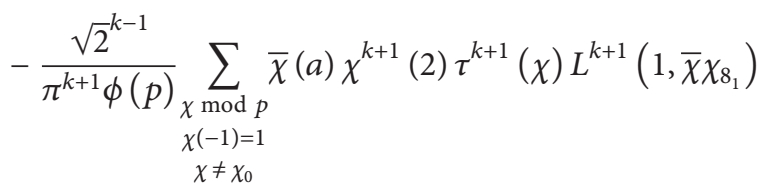

$$
\begin{aligned}
& -\frac{1}{2(i \pi)^{k+1} \phi(p)} \sum_{\substack{\chi \bmod _{\chi} \\
\chi(-1)=-1}} \bar{\chi}(a) \tau^{k+1}(\chi) \\
& \times\left((2 \chi(2)-1) L(1, \bar{\chi})-\sqrt{2} \chi(2) L\left(1, \bar{\chi} \chi_{8_{2}}\right)\right)^{k+1} .
\end{aligned}
$$

Noting the definition of $E_{1 / 4}(a, k, p)$, we can immediately get Lemma 4.

Lemma 5. Let $q \geq 3$ be an odd integer and a coprime to $q$. Then, for any positive integer $k$ with $(q, k(k+1))=1$, one has

$$
\begin{gathered}
\left|\sum_{\substack{\chi \bmod q \\
\chi(-1)=-1}}^{*} \bar{\chi}(a) \tau^{k+1}(\chi) L^{k+1}(1, \bar{\chi})\right| \\
\ll 2^{k^{2}+2 k+1} q^{k / 2} \phi(q)(2 k)^{\omega(q)} \ln ^{k+1} q, \\
\mid \begin{array}{l}
\sum_{\chi \bmod q}^{*} \bar{\chi}(a) \tau^{k+1}(\chi) L^{k+1}\left(1, \bar{\chi} \chi_{4}\right) \\
\chi(-1)=1
\end{array} \\
\mid \ll 2^{k^{2}+2 k+1} q^{k / 2} \phi(q)(2 k)^{\omega(q)} \ln ^{k+1} q, \\
\left|\begin{array}{l}
\sum_{\chi \bmod q}^{*} \bar{\chi}(a) \tau^{k+1}(\chi) L^{k+1}\left(1, \bar{\chi} \chi_{8_{1}}\right. \\
\chi(-1)=1
\end{array}\right|
\end{gathered}
$$




$$
\begin{gathered}
\ll 2^{k^{2}+2 k+1} q^{k / 2} \phi(q)(2 k)^{\omega(q)} \ln ^{k+1} q, \\
\left|\sum_{\substack{\chi \bmod q \\
\chi(-1)=-1}} \bar{\chi}(a) \tau^{k+1}(\chi) L^{k+1}\left(1, \bar{\chi} \chi_{8_{2}}\right)\right| \\
\ll 2^{k^{2}+2 k+1} q^{k / 2} \phi(q)(2 k)^{\omega(q)} \ln ^{k+1} q .
\end{gathered}
$$

Proof. Using the similar method as proving Lemma 5 of [9], we can obtain these estimates.

Lemma 6. Let $q$ and $r$ be integers with $q \geq 2$ and $(r, q)=1$, and let $\chi$ be a Dirichlet character modulo $q$. Then one has the identities

$$
\begin{gathered}
\sum_{\chi \bmod q}^{*} \chi(r)=\sum_{d \mid(q, r-1)} \mu\left(\frac{q}{d}\right) \phi(d), \\
J(q)=\sum_{d \mid q} \mu(d) \phi\left(\frac{q}{d}\right),
\end{gathered}
$$

where $\sum_{\chi \bmod q}^{*}$ denotes the summation over all primitive characters modulo $q$ and $J(q)$ is the number of primitive characters modulo $q$.

Proof. This is Lemma 3 of [10].

Lemma 7. Let $q, m$ be nonnegative integers with $q \geq 3$ an odd integer, and let $\chi$ be a Dirichlet character modulo $q$. Then, for any positive integer $k$, one has

$$
\begin{aligned}
& \sum_{\chi(-1)=-1}^{*} \chi\left(2^{m}\right)|L(1, \bar{\chi})|^{2 k} \\
& =\frac{J(q)}{2^{m+1}} \sum_{n=1}^{\infty} \frac{d_{k}\left(2^{m} n\right) d_{k}(n)}{n^{2}}+O\left(q^{\epsilon}\right), \\
& (n, q)=1 \\
& \sum_{\chi(-1)=-1}^{*} \bar{\chi}\left(2^{m}\right)|L(1, \bar{\chi})|^{2 k} \\
& =\frac{J(q)}{2^{m+1}} \sum_{n=1}^{\infty} \frac{d_{k}\left(2^{m} n\right) d_{k}(n)}{n^{2}}+O\left(q^{\epsilon}\right), \\
& (n, q)=1 \\
& \sum_{\chi(-1)=1}^{*}\left|L\left(1, \bar{\chi} \chi_{4}\right)\right|^{2 k} \\
& =\frac{J(q)}{2} \sum_{\substack{n=1 \\
(n, 2 q)=1}}^{\infty} \frac{d_{k}^{2}(n)}{n^{2}}+O\left(q^{\epsilon}\right), \\
& \sum_{\chi(-1)=1}^{*}\left|L\left(1, \bar{\chi} \chi_{8_{1}}\right)\right|^{2 k}
\end{aligned}
$$

$$
\begin{gathered}
=J(q) \sum_{\substack{n=1 \\
(n, 2 q)=1}}^{\infty} \frac{d_{k}^{2}(n)}{n^{2}}+O\left(q^{\epsilon}\right), \\
\sum_{\chi(-1)=-1}^{*}\left|L\left(1, \bar{\chi} \chi_{8_{2}}\right)\right|^{2 k} \\
=J(q) \sum_{\substack{n=1 \\
(n, 2 q)=1}}^{\infty} \frac{d_{k}^{2}(n)}{n^{2}}+O\left(q^{\epsilon}\right) .
\end{gathered}
$$

Proof. Using the similar method as proving Lemma 4 of [11], we can get the results.

Lemma 8. Let $q \geq 3$ be an odd integer, and let $k \geq 2$ be a positive integer. Then, for any fixed nonnegative integers $m, j$ such that $1 \leq j \leq k$, one has

$$
\begin{aligned}
& \sum_{\chi(-1)=-1}^{*} \chi\left(2^{m}\right) L^{k}\left(1, \bar{\chi} \chi_{8_{2}}\right) L^{j}(1, \chi) L^{k-j}\left(1, \chi \chi_{8_{2}}\right) \\
& =\frac{J(q)}{2^{m+1}} \sum_{n=1}^{\infty} \frac{\chi_{8_{2}}\left(2^{m} n\right) d_{k}\left(2^{m} n\right) r_{j}(n)}{n^{2}}+O\left(q^{\epsilon}\right), \\
& (n, q)=1 \\
& \sum_{\chi(-1)=-1}^{*} \bar{\chi}\left(2^{m}\right) L^{k}\left(1, \chi \chi_{8_{2}}\right) L^{j}(1, \bar{\chi}) L^{k-j}\left(1, \bar{\chi} \chi_{8_{2}}\right) \\
& =\frac{J(q)}{2^{m+1}} \sum_{\substack{n=1 \\
(n, q)=1}}^{\infty} \frac{\chi_{8_{2}}\left(2^{m} n\right) d_{k}\left(2^{m} n\right) r_{j}(n)}{n^{2}}+O\left(q^{\epsilon}\right), \\
& \sum_{\chi(-1)=1}^{*} \bar{\chi}\left(2^{k}\right) L^{k}\left(1, \bar{\chi} \chi_{4}\right) L^{k}\left(1, \chi \chi_{8_{1}}\right)=O\left(q^{\epsilon}\right), \\
& \sum_{\chi(-1)=1}^{*} \chi\left(2^{k}\right) L^{k}\left(1, \chi \chi_{4}\right) L^{k}\left(1, \bar{\chi} \chi_{8_{1}}\right)=O\left(q^{\epsilon}\right), \\
& \sum_{\chi(-1)=-1}^{*} \bar{\chi}\left(2^{m}\right) L^{k}(1, \bar{\chi}) L^{k}\left(1, \chi \chi_{8_{2}}\right) \\
& =\frac{J(q)}{2^{m+1}} \sum_{\substack{n=1 \\
(n, q)=1}}^{\infty} \frac{\chi_{8_{2}}\left(2^{m} n\right) d_{k}\left(2^{m} n\right) d_{k}(n)}{n^{2}}+O\left(q^{\epsilon}\right), \\
& \sum_{\chi(-1)=-1}^{*} \chi\left(2^{m}\right) L^{k}(1, \chi) L^{k}\left(1, \bar{\chi} \chi_{8_{2}}\right) \\
& =\frac{J(q)}{2^{m+1}} \sum_{\substack{n=1 \\
(n, q)=1}}^{\infty} \frac{\chi_{8_{2}}\left(2^{m} n\right) d_{k}\left(2^{m} n\right) d_{k}(n)}{n^{2}}+O\left(q^{\epsilon}\right), \\
& \sum_{\chi(-1)=-1}^{*} \bar{\chi}\left(2^{m}\right) L^{k}(1, \bar{\chi}) L^{j}(1, \chi) L^{k-j}\left(1, \chi \chi_{8_{2}}\right) \\
& =\frac{J(q)}{2^{m+1}} \sum_{\substack{n=1 \\
(n, q)=1}}^{\infty} \frac{d_{k}(n) r_{j}\left(2^{m} n\right)}{n^{2}}+O\left(q^{\epsilon}\right),
\end{aligned}
$$




$$
\begin{aligned}
& \sum_{\chi(-1)=-1}^{*} \chi\left(2^{m}\right) L^{k}(1, \chi) L^{j}(1, \bar{\chi}) L^{k-j}\left(1, \bar{\chi} \chi_{8_{2}}\right) \\
& =\frac{J(q)}{2^{m+1}} \sum_{\substack{n=1 \\
(n, q)=1}}^{\infty} \frac{d_{k}(n) r_{j}\left(2^{m} n\right)}{n^{2}}+O\left(q^{\epsilon}\right), \\
& \sum_{\chi(-1)=-1}^{*} \chi\left(2^{m}\right) L^{k}(1, \bar{\chi}) L^{j}(1, \chi) L^{k-j}\left(1, \chi \chi_{8_{2}}\right) \\
& =\frac{J(q)}{2^{m+1}} \sum_{n=1}^{\infty} \frac{d_{k}\left(2^{m} n\right) r_{j}(n)}{n^{2}}+O\left(q^{\epsilon}\right), \\
& (n, q)=1 \\
& \sum_{\chi(-1)=-1}^{*} \bar{\chi}\left(2^{m}\right) L^{k}(1, \chi) L^{j}(1, \bar{\chi}) L^{k-j}\left(1, \bar{\chi} \chi_{8_{2}}\right) \\
& =\frac{J(q)}{2^{m+1}} \sum_{\substack{n=1 \\
(n, q)=1}}^{\infty} \frac{d_{k}\left(2^{m} n\right) r_{j}(n)}{n^{2}}+O\left(q^{\epsilon}\right),
\end{aligned}
$$

where $r_{j}(n)=\sum_{t \mid n} d_{j}(t) d_{k-j}(n / t) \chi_{8_{2}}(n / t)$.

Proof. We only prove the first formula; the others can be obtained by the similar method.

For convenience, we put

$$
A(y, \chi)=\sum_{N<n \leq y} \chi(n) d_{k}(n), \quad B(y, \chi)=\sum_{N<n \leq y} \chi(n) r_{j}(n),
$$

where $N$ is a parameter with $q \leq N<q^{2 k+1}$. Then from Abel's identity we have

$$
\begin{aligned}
& L^{j}(1, \chi) L^{k-j}\left(1, \chi \chi_{8_{2}}\right) \\
& \quad=\sum_{n_{1}=1}^{\infty} \frac{\chi\left(n_{1}\right) d_{j}\left(n_{1}\right)}{n_{1}} \sum_{n_{2}=1}^{\infty} \frac{\chi \chi_{8_{2}}\left(n_{2}\right) d_{k-j}\left(n_{2}\right)}{n_{2}} \\
& \quad=\sum_{n=1}^{\infty} \frac{\chi(n) r_{j}(n)}{n}=\sum_{1 \leq n \leq N} \frac{\chi(n) r_{j}(n)}{n}+\int_{N}^{\infty} \frac{B(y, \chi)}{y^{2}} d y .
\end{aligned}
$$

Hence, we have

$$
\begin{aligned}
\sum_{\chi(-1)=-1}^{*} \chi\left(2^{m}\right) L^{k}\left(1, \bar{\chi} \chi_{8_{2}}\right) L^{j}(1, \chi) L^{k-j}\left(1, \chi \chi_{8_{2}}\right) \\
=\sum_{\chi(-1)=-1}^{*} \chi\left(2^{m}\right) \\
\quad \times\left(\sum_{1 \leq n_{1} \leq N} \frac{\bar{\chi} \chi_{8_{2}}\left(n_{1}\right) d_{k}\left(n_{1}\right)}{n_{1}}+\int_{N}^{\infty} \frac{A\left(y, \bar{\chi} \chi_{8_{2}}\right)}{y^{2}} d y\right) \\
\quad \times\left(\sum_{1 \leq n_{2} \leq N} \frac{\chi\left(n_{2}\right) r_{j}\left(n_{2}\right)}{n_{2}}+\int_{N}^{\infty} \frac{B(y, \chi)}{y^{2}} d y\right) .
\end{aligned}
$$

From the proof of Lemma 6 of [11], we know that only the term which does not contain the infinite integral will make contribution to the main term. That is,

$$
\begin{aligned}
& \sum_{\chi(-1)=-1}^{*} \chi\left(2^{m}\right) L^{k}\left(1, \bar{\chi} \chi_{8_{2}}\right) L^{j}(1, \chi) L^{k-j}\left(1, \chi \chi_{8_{2}}\right) \\
& =\sum_{\chi(-1)=-1}^{*} \chi\left(2^{m}\right) \sum_{1 \leq n_{1} \leq N 1 \leq n_{2} \leq N} \frac{\chi\left(\bar{n}_{1} n_{2}\right) \chi_{8_{2}}\left(n_{1}\right) d_{k}\left(n_{1}\right) r_{j}\left(n_{2}\right)}{n_{1} n_{2}} \\
& \quad+O\left(q^{\epsilon}\right) .
\end{aligned}
$$

Then from Lemma 6, we can write

$$
\begin{aligned}
& \sum_{\chi(-1)=-1}^{*} \chi\left(2^{m}\right) \sum_{1 \leq n_{1} \leq N} \sum_{1 \leq n_{2} \leq N} \frac{\chi\left(\bar{n}_{1} n_{2}\right) \chi_{8_{2}}\left(n_{1}\right) d_{k}\left(n_{1}\right) r_{j}\left(n_{2}\right)}{n_{1} n_{2}} \\
& =\frac{1}{2} \sum_{1 \leq n_{1} \leq N} \sum_{1 \leq n_{2} \leq N} \frac{\chi_{8_{2}}\left(n_{1}\right) d_{k}\left(n_{1}\right) r_{j}\left(n_{2}\right)}{n_{1} n_{2}} \\
& \times \sum_{d \mid\left(q, \bar{n}_{1} 2^{m} n_{2}-1\right)} \mu(d) \phi\left(\frac{q}{d}\right) \\
& -\frac{1}{2} \sum_{1 \leq n_{1} \leq N} \sum_{1 \leq n_{2} \leq N}^{\prime} \frac{\chi_{8_{2}}\left(n_{1}\right) d_{k}\left(n_{1}\right) r_{j}\left(n_{2}\right)}{n_{1} n_{2}} \\
& \times \sum_{d \mid\left(q, \bar{n}_{1} 2^{m} n_{2}+1\right)} \mu(d) \phi\left(\frac{q}{d}\right) \\
& =\frac{1}{2} \sum_{d \mid q} \mu(d) \phi\left(\frac{q}{d}\right) \sum_{\substack{1 \leq n_{1} \leq N \\
\bar{n}_{1} 2^{m} n_{2} \equiv 1(\bmod d)}} \sum_{\substack{1 \leq n_{2} \leq N \\
\prime}} \frac{\chi_{8_{2}}\left(n_{1}\right) d_{k}\left(n_{1}\right) r_{j}\left(n_{2}\right)}{n_{1} n_{2}} \\
& -\frac{1}{2} \sum_{d \mid q} \mu(d) \phi\left(\frac{q}{d}\right) \\
& \times \sum_{\substack{1 \leq n_{1} \leq N \\
\bar{n}_{1} 2^{m} n_{2} \equiv-1(\bmod d)}} \sum_{\substack{1 \leq n_{2} \leq N \\
n_{1} n_{2}}} \frac{\chi_{8_{2}}\left(n_{1}\right) d_{k}\left(n_{1}\right) r_{j}\left(n_{2}\right)}{n_{1}}
\end{aligned}
$$

where $\sum_{1 \leq n \leq N}^{\prime}$ denotes the summation over all integers $n$ with $(n, q)=1$ and $1 \leq n \leq N$. Now we split the above first sum into the following four cases:

(i) $d \leq n_{1} \leq N$ and $d / 2^{m} \leq n_{2} \leq N$;

(ii) $d \leq n_{1} \leq N$ and $1 \leq n_{2} \leq d / 2^{m}-1$;

(iii) $1 \leq n_{1} \leq d-1$ and $d / 2^{m} \leq n_{2} \leq N$;

(iv) $1 \leq n_{1} \leq d-1$ and $1 \leq n_{2} \leq d / 2^{m}-1$. 
By using the similar method as proving Lemma 6 of [11], we know that the main term will be

$$
\begin{aligned}
\frac{1}{2} \sum_{d \mid q} \mu(d) \phi\left(\frac{q}{d}\right) \sum_{\substack{1 \leq n_{1} \leq N \\
\bar{n}_{1} 2^{m} n_{2} \equiv 1(\bmod d)}} \sum_{1 \leq n_{2} \leq N} \frac{\chi_{8_{2}}\left(n_{1}\right) d_{k}\left(n_{1}\right) r_{j}\left(n_{2}\right)}{n_{1} n_{2}} \\
=\frac{1}{2} \sum_{d \mid q} \mu(d) \phi\left(\frac{q}{d}\right) \\
\quad \times \sum_{\substack{n_{2}=1 \\
\left(n_{2}, q\right)=1}}^{\infty} \frac{\chi_{8_{2}}\left(2^{m} n_{2}\right) d_{k}\left(2^{m} n_{2}\right) r_{j}\left(n_{2}\right)}{2^{m} n_{2}^{2}}+O\left(q^{\epsilon}\right) .
\end{aligned}
$$

Hence we have

$$
\begin{aligned}
& \sum_{\chi(-1)=-1}^{*} \chi\left(2^{m}\right) L^{k}\left(1, \bar{\chi} \chi_{8_{2}}\right) L^{j}(1, \chi) L^{k-j}\left(1, \chi \chi_{8_{2}}\right) \\
& \quad=\frac{J(q)}{2^{m+1}} \sum_{\substack{n=1 \\
(n, q)=1}}^{\infty} \frac{\chi_{8_{2}}\left(2^{m} n\right) d_{k}\left(2^{m} n\right) r_{j}(n)}{n^{2}}+O\left(q^{\epsilon}\right) .
\end{aligned}
$$

This proves Lemma 8 .

Lemma 9. Let $q \geq 3$ be an odd integer and let $k \geq 2$ be a positive integer. Then, for any fixed nonnegative integers $a, b \leq$ $k$ such that $a \neq 0$ while $b=0$, and $a \neq k$ while $b=k$, one has

$$
\begin{aligned}
& \sum_{\substack{\chi \bmod q \\
\chi(-1)=-1}}^{*} \bar{\chi}\left(2^{a}\right) \chi\left(2^{b}\right) L^{a}(1, \bar{\chi}) L^{b}(1, \chi) \\
& \quad \times L^{k-a}\left(1, \bar{\chi} \chi_{8_{2}}\right) L^{k-b}\left(1, \chi \chi_{8_{2}}\right) \\
& =\left\{\begin{array}{l}
\frac{J(q)}{2^{b-a+1}} \sum_{\substack{n=1 \\
(n, q)=1}}^{\infty} \frac{r_{a}\left(2^{b-a} n\right) r_{b}(n)}{n^{2}}+O\left(q^{\epsilon}\right) \text { if } a \leq b ; \\
\frac{J(q)}{2^{a-b+1}} \sum_{\substack{n=1 \\
(n, q)=1}}^{\infty} \frac{r_{a}(n) r_{b}\left(2^{a-b} n\right)}{n^{2}}+O\left(q^{\epsilon}\right) \quad \text { if } a>b .
\end{array}\right.
\end{aligned}
$$

Proof. Using the similar method as proving Lemma 6 of [11], we can get the results.

\section{Proof of Theorems}

In this section, we will complete the proof of our theorems. From Lemmas 4 and 5, we can immediately get the result of Theorem 1.

Now we come to prove Theorem 2. Noting that

$$
\sum_{a=1}^{p} \chi(a)=0
$$

if $\chi \neq \chi_{0}$, from Lemma 4 and the orthogonality of Dirichlet character, we can write

$$
\begin{aligned}
& \sum_{a=1}^{p-1}\left|E_{1 / 4}(a, k, p)\right|^{2} \\
& =\frac{1}{4 \pi^{2 k+2} \phi(p)} \sum_{\substack{\chi \bmod p \\
\chi(-1)=1}}^{*}|\tau(\chi)|^{2 k+2}\left|L\left(1, \bar{\chi} \chi_{4}\right)\right|^{2 k+2} \\
& +O\left(\frac{1}{p}\right)-\frac{2^{k-1}}{\pi^{2 k+2} \phi(p)} \sum_{\substack{\chi \bmod p \\
\chi(-1)=1}}^{*}|\tau(\chi)|^{2 k+2} \\
& \times\left|L\left(1, \bar{\chi} \chi_{8_{1}}\right)\right|^{2 k+2} \\
& +\frac{1}{2^{2 k+4} \pi^{2 k+2} \phi(p)} \\
& \times \sum_{\substack{\chi \bmod p \\
\chi(-1)=-1}}^{*}|2+\bar{\chi}(2)-\bar{\chi}(4)|^{2 k+2}|\tau(\chi)|^{2 k+2}|L(1, \bar{\chi})|^{2 k+2} \\
& -\frac{(-1)^{k+1}}{4 \pi^{2 k+2} \phi(p)} \sum_{\substack{\chi \bmod p \\
\chi(-1)=-1}}^{*}|\tau(\chi)|^{2 k+2} \\
& \times\left|(2 \chi(2)-1) L(1, \bar{\chi})-\sqrt{2} \chi(2) L\left(1, \bar{\chi} \chi_{8_{2}}\right)\right|^{2 k+2} \\
& -\frac{\sqrt{2}^{k-3}}{\pi^{2 k+2} \phi(p)} \sum_{\substack{\chi \bmod p \\
\chi(-1)=1}}^{*} \bar{\chi}\left(2^{k+1}\right)|\tau(\chi)|^{2 k+2} \\
& \times L^{k+1}\left(1, \bar{\chi} \chi_{4}\right) L^{k+1}\left(1, \chi \chi_{8_{1}}\right) \\
& -\frac{\sqrt{2^{k-3}}}{\pi^{2 k+2} \phi(p)} \sum_{\substack{\chi \bmod p \\
\chi(-1)=1}}^{*} \chi\left(2^{k+1}\right)|\tau(\chi)|^{2 k+2} \\
& \times L^{k+1}\left(1, \chi \chi_{4}\right) L^{k+1}\left(1, \bar{\chi} \chi_{8_{1}}\right) \\
& -\frac{(-1)^{k+1}}{2^{k+3} \pi^{2 k+2} \phi(p)} \sum_{\substack{\chi \bmod p \\
\chi(-1)=-1}}^{*}|\tau(\chi)|^{2 k+2} \\
& \times(2+\bar{\chi}(2)-\bar{\chi}(4))^{k+1} L^{k+1}(1, \bar{\chi}) \\
& \times\left((2 \bar{\chi}(2)-1) L(1, \chi)-\sqrt{2} \bar{\chi}(2) L\left(1, \chi \chi_{8_{2}}\right)\right)^{k+1} \\
& -\frac{(-1)^{k+1}}{2^{k+3} \pi^{2 k+2} \phi(p)} \sum_{\substack{\chi \bmod p \\
\chi(-1)=-1}}^{*}|\tau(\chi)|^{2 k+2} \\
& \times(2+\chi(2)-\chi(4))^{k+1} L^{k+1}(1, \chi) \\
& \times\left((2 \chi(2)-1) L(1, \bar{\chi})-\sqrt{2} \chi(2) L\left(1, \bar{\chi} \chi_{8_{2}}\right)\right)^{k+1}
\end{aligned}
$$




$$
\begin{aligned}
& =\frac{p^{k+1}}{4 \pi^{2 k+2} \phi(p)} \sum_{\substack{\chi \bmod p \\
\chi(-1)=1}}^{*}\left|L\left(1, \bar{\chi} \chi_{4}\right)\right|^{2 k+2} \\
& -\frac{2^{k-1} p^{k+1}}{\pi^{2 k+2} \phi(p)} \sum_{\substack{\chi \bmod p \\
\chi(-1)=1}}^{*}\left|L\left(1, \bar{\chi} \chi_{8_{1}}\right)\right|^{2 k+2} \\
& +\frac{p^{k+1}}{2^{2 k+4} \pi^{2 k+2} \phi(p)} \sum_{i=0}^{k+1}(-2)^{k+1-i} C_{k+1}^{i} \\
& \times \sum_{j=0}^{i} 6^{j} \sum_{s=0}^{i-j k+1-i} \sum_{t=0}^{s} C_{i-j}^{t} C_{k+1-i} \\
& \times \sum_{\substack{\chi \bmod p \\
\chi(-1)=-1}}^{*} \chi\left(2^{2 s+4 t+i+j-2 k-2}\right)|L(1, \bar{\chi})|^{2 k+2} \\
& -\frac{(-p)^{k+1}}{4 \pi^{2 k+2} \phi(p)} \sum_{j_{1}=0}^{k+1} \sum_{j_{2}=0}^{k+1} \sum_{l_{1}=0 l_{2}=0}^{j_{1}} \sum_{j_{2}}^{j_{2}}(-1)^{l_{1}+l_{2}} \sqrt{2}^{2 k+2\left(l_{1}+l_{2}\right)+2-\left(j_{1}+j_{2}\right)} \\
& \times C_{k+1}^{j_{1}} C_{k+1}^{j_{2}} C_{j_{1}}^{l_{1}} C_{j_{2}}^{l_{2}} \sum_{\substack{\chi \bmod p \\
\chi(-1)=-1}}^{*} \bar{\chi}\left(2^{j_{1}-l_{1}}\right) \chi\left(2^{j_{2}-l_{2}}\right) L^{j_{1}}(1, \bar{\chi}) \\
& \times L^{j_{2}}(1, \chi) L^{k+1-j_{1}}\left(1, \bar{\chi} \chi_{8_{2}}\right) L^{k+1-j_{2}}\left(1, \chi \chi_{8_{2}}\right) \\
& -\frac{\sqrt{2}^{k-3} p^{k+1}}{\pi^{2 k+2} \phi(p)} \sum_{\chi \bmod p}^{*} \bar{\chi}\left(2^{k+1}\right) L^{k+1}\left(1, \bar{\chi} \chi_{4}\right) L^{k+1}\left(1, \chi \chi_{8_{1}}\right) \\
& \chi(-1)=1 \\
& -\frac{\sqrt{2}^{k-3} p^{k+1}}{\pi^{2 k+2} \phi(p)} \sum_{\substack{\chi \bmod p \\
\chi(-1)=1}}^{*} \chi\left(2^{k+1}\right) L^{k+1}\left(1, \chi \chi_{4}\right) L^{k+1}\left(1, \bar{\chi} \chi_{8_{1}}\right) \\
& -\frac{(-p)^{k+1}}{2^{k+3} \pi^{2 k+2} \phi(p)} \\
& \times \sum_{j_{1}=0}^{k+1} \sum_{j_{2}=0}^{k+1} \sum_{l_{1}=0 l_{2}=0}^{j_{1}} \sum_{j_{2}}^{j_{2}}(-1)^{j_{1}+l_{2}} \sqrt{2}^{k+2\left(l_{1}+l_{2}\right)+1-j_{2}} C_{k+1}^{j_{1}} C_{k+1}^{j_{2}} C_{j_{1}}^{l_{1}} C_{j_{2}}^{l_{2}} \\
& \times \sum_{\substack{\chi \bmod p \\
\chi(-1)=-1}}^{*} \bar{\chi}\left(2^{3 k+3+l_{2}-j_{1}-j_{2}-l_{1}}\right) \\
& \times L^{k+1}(1, \bar{\chi}) L^{j_{2}}(1, \chi) L^{k+1-j_{2}}\left(1, \chi \chi_{8_{2}}\right) \\
& -\frac{(-p)^{k+1}}{2^{k+3} \pi^{2 k+2} \phi(p)} \\
& \times \sum_{j_{1}=0}^{k+1} \sum_{j_{2}=0}^{k+1} \sum_{l_{1}=0 l_{2}=0}^{j_{1}} \sum^{j_{2}}(-1)^{j_{1}+l_{2}} \sqrt{2}^{k+2\left(l_{1}+l_{2}\right)+1-j_{2}} C_{k+1}^{j_{1}} C_{k+1}^{j_{2}} C_{j_{1}}^{l_{1}} C_{j_{2}}^{l_{2}} \\
& \begin{array}{l}
\times \sum_{\substack{\chi \bmod p \\
\chi(-1)=-1}}^{*} \chi\left(2^{3 k+3+l_{2}-j_{1}-j_{2}-l_{1}}\right) \\
\times L^{k+1}(1, \chi) L^{j_{2}}(1, \bar{\chi}) L^{k+1-j_{2}}\left(1, \bar{\chi} \chi_{8_{2}}\right) .
\end{array} \\
& \sum_{j_{1}=0}^{k+1} \sum_{j_{2}=0}^{k+1} \sum_{l_{1}=0}^{j_{1}} \sum_{l_{2}=0}^{j_{2}}(-1)^{l_{1}+l_{2}} \sqrt{2}^{2 k+2\left(l_{1}+l_{2}\right)+2-\left(j_{1}+j_{2}\right)} \\
& \times C_{k+1}^{j_{1}} C_{k+1}^{j_{2}} C_{j_{1}}^{l_{1}} C_{j_{2}}^{l_{2}} \\
& \times \sum_{\substack{\chi \bmod p \\
\chi(-1)=-1}}^{*} \bar{\chi}\left(2^{j_{1}-l_{1}}\right) \times \chi\left(2^{j_{2}-l_{2}}\right) L^{j_{1}}(1, \bar{\chi}) \\
& \times L^{j_{2}}(1, \chi) L^{k+1-j_{1}}\left(1, \bar{\chi} \chi_{8_{2}}\right) L^{k+1-j_{2}}\left(1, \chi \chi_{8_{2}}\right) \\
& =2^{k+1} \sum_{\substack{\chi \bmod p \\
\chi(-1)=-1}}^{*}\left|L\left(1, \bar{\chi} \chi_{8_{2}}\right)\right|^{2 k+2} \\
& +\sum_{l_{1}=0}^{k+1} \sum_{l_{2}=0}^{k+1}(-2)^{l_{1}+l_{2}} C_{k+1}^{l_{1}} C_{k+1}^{l_{2}} \sum_{\substack{\chi \bmod p \\
\chi(-1)=-1}}^{*} \chi\left(2^{l_{1}-l_{2}}\right)|L(1, \bar{\chi})|^{2 k+2} \\
& +\sum_{j_{2}=1}^{k+1} \sum_{l_{2}=0}^{j_{2}}(-1)^{l_{2}} \sqrt{2}^{2 k+2 l_{2}+2-j_{2}} C_{k+1}^{j_{2}} C_{j_{2}}^{l_{2}} \\
& \times \sum_{\substack{\chi \bmod p \\
\chi(-1)=-1}}^{*} \chi\left(2^{j_{2}-l_{2}}\right) L^{j_{2}}(1, \chi) \\
& \times L^{k+1}\left(1, \bar{\chi} \chi_{8_{2}}\right) L^{k+1-j_{2}}\left(1, \chi \chi_{8_{2}}\right) \\
& +\sum_{j_{1}=1}^{k+1} \sum_{l_{1}=0}^{j_{1}}(-1)^{l_{1}} \sqrt{2}^{2 k+2 l_{1}+2-j_{1}} C_{k+1}^{j_{1}} C_{j_{1}}^{l_{1}} \\
& \times \sum_{\substack{\chi \bmod p \\
\chi(-1)=-1}}^{*} \bar{\chi}\left(2^{j_{1}-l_{1}}\right) L^{j_{1}}(1, \bar{\chi}) \\
& \times L^{k+1-j_{1}}\left(1, \bar{\chi} \chi_{8_{2}}\right) L^{k+1}\left(1, \chi \chi_{8_{2}}\right) \\
& +\sum_{j_{2}=0}^{k} \sum_{l_{1}=0}^{k+1} \sum_{l_{2}=0}^{j_{2}}(-1)^{l_{1}+l_{2}} \sqrt{2}^{k+2\left(l_{1}+l_{2}\right)+1-j_{2}} C_{k+1}^{j_{2}} C_{k+1}^{l_{1}} C_{j_{2}}^{l_{2}} \\
& \times \sum_{\substack{\chi \bmod p \\
\chi(-1)=-1}}^{*} \chi\left(2^{\left(j_{2}-l_{2}\right)-\left(k+1-l_{1}\right)}\right) L^{k+1}(1, \bar{\chi}) \\
& \times L^{j_{2}}(1, \chi) L^{k+1-j_{2}}\left(1, \chi \chi_{8_{2}}\right) \\
& +\sum_{j_{1}=0}^{k} \sum_{l_{1}=0}^{j_{1}} \sum_{l_{2}=0}^{k+1}(-1)^{l_{1}+l_{2}} \sqrt{2}^{k+2\left(l_{1}+l_{2}\right)+1-j_{1}} C_{k+1}^{j_{1}} C_{j_{1}}^{l_{1}} C_{k+1}^{l_{2}}
\end{aligned}
$$




$$
\begin{aligned}
& \times \sum_{\substack{\chi \bmod p \\
\chi(-1)=-1}}^{*} \chi\left(2^{\left(k+1-l_{2}\right)-\left(j_{1}-l_{1}\right)}\right) L^{j_{1}}(1, \bar{\chi}) \\
& \times L^{k+1}(1, \chi) L^{k+1-j_{1}}\left(1, \bar{\chi} \chi_{8_{2}}\right) \\
& +\sum_{j_{1}=1}^{k} \sum_{j_{2}=1}^{k} \sum_{l_{1}=0}^{j_{1}} \sum_{l_{2}=0}^{j_{2}}(-1)^{l_{1}+l_{2}} \sqrt{2}^{2 k+2\left(l_{1}+l_{2}\right)+2-\left(j_{1}+j_{2}\right)} \\
& \times C_{k+1}^{j_{1}} C_{k+1}^{j_{2}} C_{j_{1}}^{l_{1}} C_{j_{2}}^{l_{2}} \sum_{\substack{\chi \bmod p \\
\chi(-1)=-1}}^{*} \bar{\chi}\left(2^{j_{1}-l_{1}}\right) \times \chi\left(2^{j_{2}-l_{2}}\right) L^{j_{1}}(1, \bar{\chi}) \\
& \times L^{j_{2}}(1, \chi) L^{k+1-j_{1}}\left(1, \bar{\chi} \chi_{8_{2}}\right) L^{k+1-j_{2}}\left(1, \chi \chi_{8_{2}}\right), \\
& \sum_{j_{1}=0}^{k+1} \sum_{j_{2}=0}^{k+1} \sum_{l_{1}=0}^{j_{1}} \sum_{l_{2}=0}^{j_{2}}(-1)^{j_{1}+l_{2}} \sqrt{2}^{k+2\left(l_{1}+l_{2}\right)+1-j_{2}} C_{k+1}^{j_{1}} C_{k+1}^{j_{2}} C_{j_{1}}^{l_{1}} C_{j_{2}}^{l_{2}} \\
& \times \sum_{\substack{\chi \bmod p \\
\chi(-1)=-1}}^{*} \bar{\chi}\left(2^{3 k+3+l_{2}-j_{1}-j_{2}-l_{1}}\right) \\
& \times L^{k+1}(1, \bar{\chi}) L^{j_{2}}(1, \chi) L^{k+1-j_{2}}\left(1, \chi \chi_{8_{2}}\right) \\
& =\sum_{j_{1}=0}^{k+1} \sum_{l_{1}=0}^{j_{1}}(-1)^{j_{1}} \sqrt{2}^{k+2 l_{1}+1} C_{k+1}^{j_{1}} C_{j_{1}}^{l_{1}} \\
& \times \sum_{\substack{\chi \bmod p \\
\chi(-1)=-1}}^{*} \bar{\chi}\left(2^{3 k+3-j_{1}-l_{1}}\right) L^{k+1}(1, \bar{\chi}) L^{k+1}\left(1, \chi \chi_{8_{2}}\right) \\
& +\sum_{j_{1}=0}^{k+1} \sum_{l_{1}=0}^{j_{1}} \sum_{l_{2}=0}^{k+1}(-1)^{j_{1}+l_{2}} 2^{l_{1}+l_{2}} C_{k+1}^{j_{1}} C_{j_{1}}^{l_{1}} C_{k+1}^{l_{2}} \\
& \times \sum_{\substack{\chi \bmod p \\
\chi(-1)=-1}}^{*} \bar{\chi}\left(2^{2 k+2+l_{2}-j_{1}-l_{1}}\right)|L(1, \chi)|^{2 k+2} \\
& +\sum_{j_{1}=0}^{k+1} \sum_{j_{2}=1}^{k} \sum_{l_{1}=0}^{j_{1}} \sum_{l_{2}=0}^{j_{2}}(-1)^{j_{1}+l_{2}} \sqrt{2}^{k+2\left(l_{1}+l_{2}\right)+1-j_{2}} \\
& \times C_{k+1}^{j_{1}} C_{k+1}^{j_{2}} C_{j_{1}}^{l_{1}} C_{j_{2}}^{l_{2}} \sum_{\substack{\chi \bmod p \\
\chi(-1)=-1}}^{*} \bar{\chi}\left(2^{3 k+3+l_{2}-j_{1}-j_{2}-l_{1}}\right) \\
& \times L^{k+1}(1, \bar{\chi}) L^{j_{2}}(1, \chi) L^{k+1-j_{2}}\left(1, \chi \chi_{8_{2}}\right) .
\end{aligned}
$$

Combining the above with Lemmas 7-9, we have

$$
\sum_{a=1}^{p-1}\left|E_{1 / 4}(a, k, p)\right|^{2}=\frac{A(k)}{\pi^{2 k+2}} p^{k+1}+O_{k}\left(p^{k+\epsilon}\right),
$$

where

$$
\begin{aligned}
& A(k)=\left(\frac{1}{8}-2^{k-1}+(-1)^{k} 2^{k-1}\right) \sum_{\substack{n=1 \\
(n, 2 p)=1}}^{\infty} \frac{d_{k+1}^{2}(n)}{n^{2}} \\
& +\sum_{i=0}^{k+1} C_{k+1}^{i} \sum_{j=0}^{i} 6^{j} \sum_{s=0}^{i-j} \sum_{t=0}^{k+1-i} C_{i-j}^{s} C_{k+1-i}^{t} \\
& \times \frac{(-1)^{k+1-i}}{2^{|2 s+4 t+i+j-2 k-2|+k+i+5}} \\
& \times \sum_{\substack{n=1 \\
(n, p)=1}}^{\infty} \frac{d_{k+1}\left(2^{|2 s+4 t+i+j-2 k-2|} n\right) d_{k+1}(n)}{n^{2}} \\
& +\sum_{j_{1}=0}^{k+1} \sum_{l_{1}=0}^{j_{1}} \sum_{l_{2}=0}^{k+1} C_{k+1}^{j_{1}} C_{j_{1}}^{l_{1}} C_{k+1}^{l_{2}} \frac{(-1)^{k+j_{1}+l_{2}}}{2^{\left|2 k+2+l_{2}-j_{1}-l_{1}\right|+k+3-l_{1}-l_{2}}} \\
& \times \sum_{\substack{n=1 \\
(n, p)=1}}^{\infty} \frac{d_{k+1}\left(2^{\left|2 k+2+l_{2}-j_{1}-l_{1}\right|} n\right) d_{k+1}(n)}{n^{2}} \\
& +\sum_{j_{1}=0}^{k+1} \sum_{j_{2}=1}^{k} \sum_{l_{1}=0}^{j_{1}} \sum_{l_{2}=0}^{j_{2}} C_{k+1}^{j_{1}} C_{k+1}^{j_{2}} C_{j_{1}}^{l_{1}} C_{j_{2}}^{l_{2}} \\
& \times(-1)^{k+j_{1}+l_{2}} \sqrt{2}^{2 j_{1}+j_{2}+4 l_{1}-7 k-11} \\
& \times \sum_{\substack{n=1 \\
(n, p)=1}}^{\infty} \frac{r_{j_{2}}\left(2^{3 k+3+l_{2}-j_{1}-j_{2}-l_{1}} n\right) d_{k+1}(n)}{n^{2}} \\
& +\sum_{j_{2}=1}^{k+1}(-1)^{k+j_{2}} \sqrt{2}^{2 k+j_{2}+2} C_{k+1}^{j_{2}} \sum_{\substack{n=1 \\
(n, p)=1}}^{\infty} \frac{d_{k+1}(n) r_{j_{2}}(n)}{n^{2}} \\
& +\sum_{l_{1}=0}^{k+1} \sum_{l_{2}=0}^{k+1} C_{k+1}^{l_{1}} C_{k+1}^{l_{2}} \frac{(-1)^{k+l_{1}+l_{2}}}{2^{\left|l_{1}-l_{2}\right|+2-l_{1}-l_{2}}} \\
& \times \sum_{\substack{n=1 \\
(n, p)=1}}^{\infty} \frac{d_{k+1}\left(2^{\left|l_{1}-l_{2}\right|} n\right) d_{k+1}(n)}{n^{2}} \\
& +\sum_{\substack{j_{2}=0 \\
j_{2}-l_{2} \geq k+1-l_{1}}}^{k} \sum_{l_{1}=0}^{k+1} \sum_{l_{2}=0}^{j_{2}}(-1)^{k+l_{1}+l_{2}} C_{k+1}^{j_{2}} C_{k+1}^{l_{1}} C_{j_{2}}^{l_{2}} \sqrt{2}^{3 k+4 l_{2}+1-3 j_{2}} \\
& \times \sum_{\substack{n=1 \\
(n, p)=1}}^{\infty} \frac{d_{k+1}\left(2^{\left(j_{2}-l_{2}\right)-\left(k+1-l_{1}\right)} n\right) r_{j_{2}}(n)}{n^{2}}
\end{aligned}
$$




$$
\begin{aligned}
& +\sum_{\substack{j_{2}=0 \\
j_{2}-l_{2}<k+1-l_{1}}}^{k} \sum_{l_{1}=0}^{k+1} \sum_{l_{2}=0}^{j_{2}}(-1)^{k+l_{1}+l_{2}} C_{k+1}^{j_{2}} C_{k+1}^{l_{1}} C_{j_{2}}^{l_{2}} \sqrt{2}^{4 l_{1}+j_{2}-k-3} \\
& \times \sum_{\substack{n=1 \\
(n, p)=1}}^{\infty} \frac{d_{k+1}(n) r_{j_{2}}\left(2^{\left(k+1-l_{1}\right)-\left(j_{2}-l_{2}\right)} n\right)}{n^{2}} \\
& +\sum_{\substack{j_{1}=0 \\
j_{1}-l_{1} \geq k+1-l_{2}}}^{k} \sum_{l_{1}=0}^{j_{1}} \sum_{l_{2}=0}^{k+1}(-1)^{k+l_{1}+l_{2}} C_{k+1}^{j_{1}} C_{j_{1}}^{l_{1}} C_{k+1}^{l_{2}} \sqrt{2}^{3 k+4 l_{1}+1-3 j_{1}} \\
& \times \sum_{\substack{n=1 \\
(n, p)=1}}^{\infty} \frac{d_{k+1}\left(2^{\left(j_{1}-l_{1}\right)-\left(k+1-l_{2}\right)} n\right) r_{j_{2}}(n)}{n^{2}} \\
& +\sum_{\substack{j_{1}=0 \\
j_{1}-l_{1}<k+1-l_{2}}}^{k} \sum_{l_{1}=0}^{j_{1}} \sum_{l_{2}=0}^{k+1}(-1)^{k+l_{1}+l_{2}} C_{k+1}^{j_{1}} C_{j_{1}}^{l_{1}} C_{k+1}^{l_{2}} \sqrt{2}^{4 l_{2}+j_{1}-k-3} \\
& \times \sum_{\substack{n=1 \\
(n, p)=1}}^{\infty} \frac{d_{k+1}(n) r_{j_{2}}\left(2^{\left(k+1-l_{2}\right)-\left(j_{1}-l_{1}\right)} n\right)}{n^{2}} \\
& +\sum_{j_{1}=1}^{k} \sum_{\substack{j_{2}=1 \\
j_{1}-l_{1} \geq j_{2}-l_{2}}}^{k} \sum_{l_{1}=0}^{j_{1}} \sum_{l_{2}=0}^{j_{2}}(-1)^{k+l_{1}+l_{2}} \\
& \times C_{k+1}^{j_{1}} C_{k+1}^{j_{2}} C_{j_{1}}^{l_{1}} C_{j_{2}}^{l_{2}} \sqrt{2}^{2 k+4 l_{1}+j_{2}-3 j_{1}} \\
& \times \sum_{\substack{n=1 \\
(n, p)=1}}^{\infty} \frac{r_{j_{1}-l_{1}}(n) r_{j_{2}-l_{2}}\left(2^{\left(j_{1}-l_{1}\right)-\left(j_{2}-l_{2}\right)} n\right)}{n^{2}} \\
& +\sum_{j_{1}=1}^{k} \sum_{\substack{j_{2}=1 \\
j_{1}-l_{1}<j_{2}-l_{2}}}^{k} \sum_{l_{1}=0}^{j_{1}} \sum_{l_{2}=0}^{j_{2}}(-1)^{k+l_{1}+l_{2}} \\
& \times C_{k+1}^{j_{1}} C_{k+1}^{j_{2}} C_{j_{1}}^{l_{1}} C_{j_{2}}^{l_{2}} \sqrt{2}^{2 k+4 l_{1}+j_{2}-3 j_{1}} \\
& \times \sum_{\substack{n=1 \\
(n, p)=1}}^{\infty} \frac{r_{j_{2}-l_{2}}(n) r_{j_{1}-l_{1}}\left(2^{\left(j_{2}-l_{2}\right)-\left(j_{1}-l_{1}\right)} n\right)}{n^{2}} .
\end{aligned}
$$

\section{Acknowledgments}

The author expresses his gratitude to the referee for his/her very helpful and detailed comments. This work is supported by the NSF (11201275) and the Natural Science Foundation of Shaanxi Province of China (no.2011JQ1010).

\section{References}

[1] R. K. Guy, Unsolved Problems in Number Theory, Springer, New York, NY, USA, 1994.

[2] W. P. Zhang, "A problem of D.H. Lehmer and its Generalization (II)," Compositio Mathematica, vol. 91, pp. 47-56, 1994.

[3] W. P. Zhang, "A problem of D.H. Lehmer and its mean square value formula," Japanese Journal of Mathematics, vol. 29, pp. 109-116, 2003.

[4] W. Zhang, X. Zongben, and Y. Yuan, "A problem of D.H. Lehmer and its mean square value formula," Journal of Number Theory, vol. 103, no. 2, pp. 197-213, 2003.

[5] Z. Xu and W. Zhang, "On a problem of D.H. Lehmer over short intervals," Journal of Mathematical Analysis and Applications, vol. 320, no. 2, pp. 756-770, 2006.

[6] Z. F. Xu and T. P. Zhang, "High-dimensional D. H. Lehmer problem over short intervals," Acta Mathematica Sinica, vol. 30, pp. 213-228, 2014.

[7] Berndt and C. Bruce, "Classical theorems on quadratic residues,' L'Enseignement Mathématique, vol. 22, pp. 261-304, 1976.

[8] G. L. Ren and W. P. Zhang, "On the mean value of a sum analogous to character sums over short intervals," Czechoslovak Mathematical Journal, vol. 133, pp. 651-668, 2008.

[9] X. Zhefeng and Z. Wenpeng, "On the order of the highdimensional Cochrane sum and its mean value," Journal of Number Theory, vol. 117, no. 1, pp. 131-145, 2006.

[10] Z. Wenpeng, "On a Cochrane sum and its hybrid mean value formula," Journal of Mathematical Analysis and Applications, vol. 267, no. 1, pp. 89-96, 2002.

[11] Z. Xu, "Mean value of the character sums over interval [1, q/8)," Taiwanese Journal of Mathematics, vol. 13, no. 1, pp. 169-187, 2009.

This proves Theorem 2 .

\section{Conflict of Interests}

The author declares that there is no conflict of interests regarding the publication of this paper. 


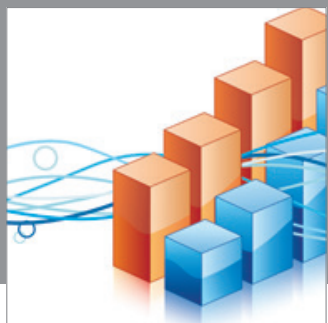

Advances in

Operations Research

mansans

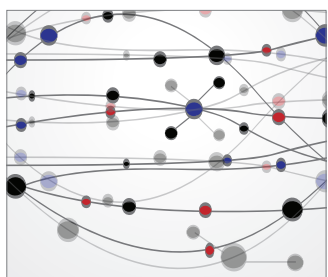

The Scientific World Journal
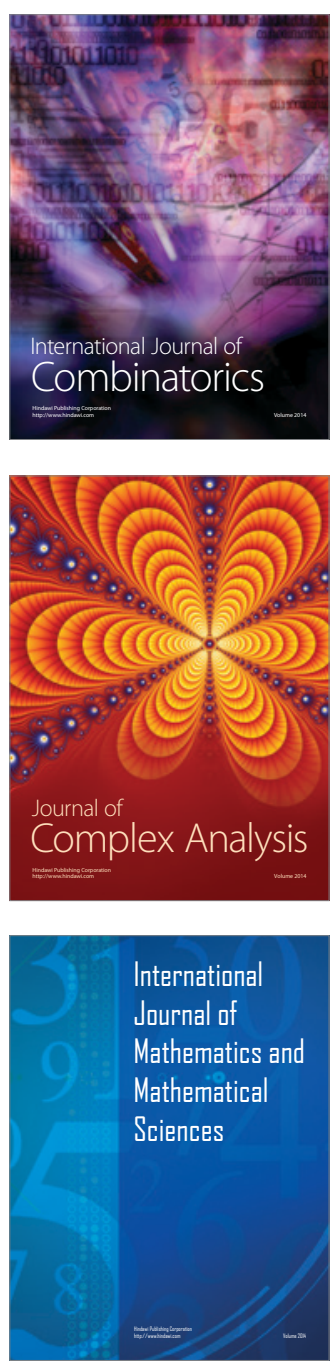
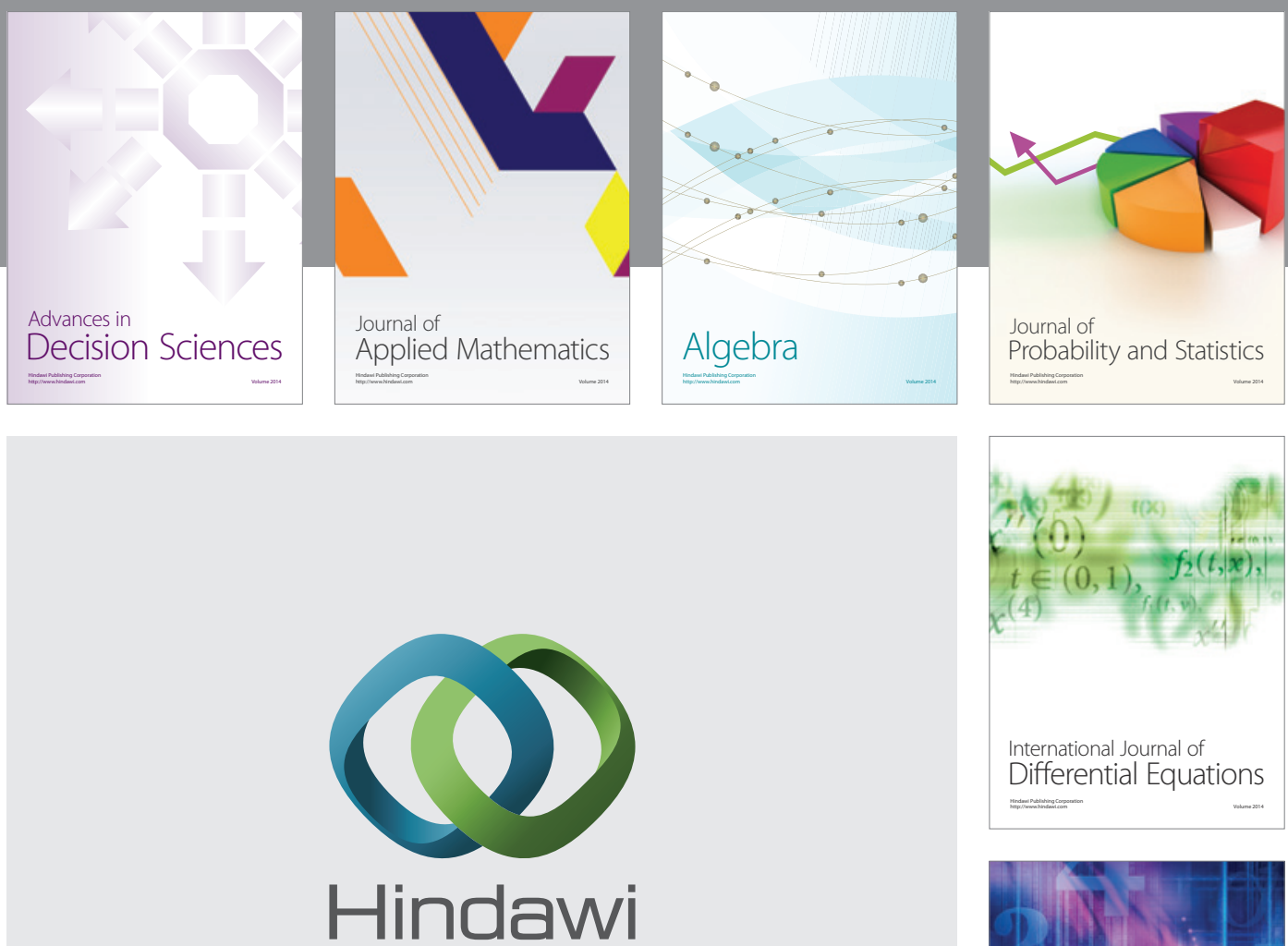

Submit your manuscripts at http://www.hindawi.com
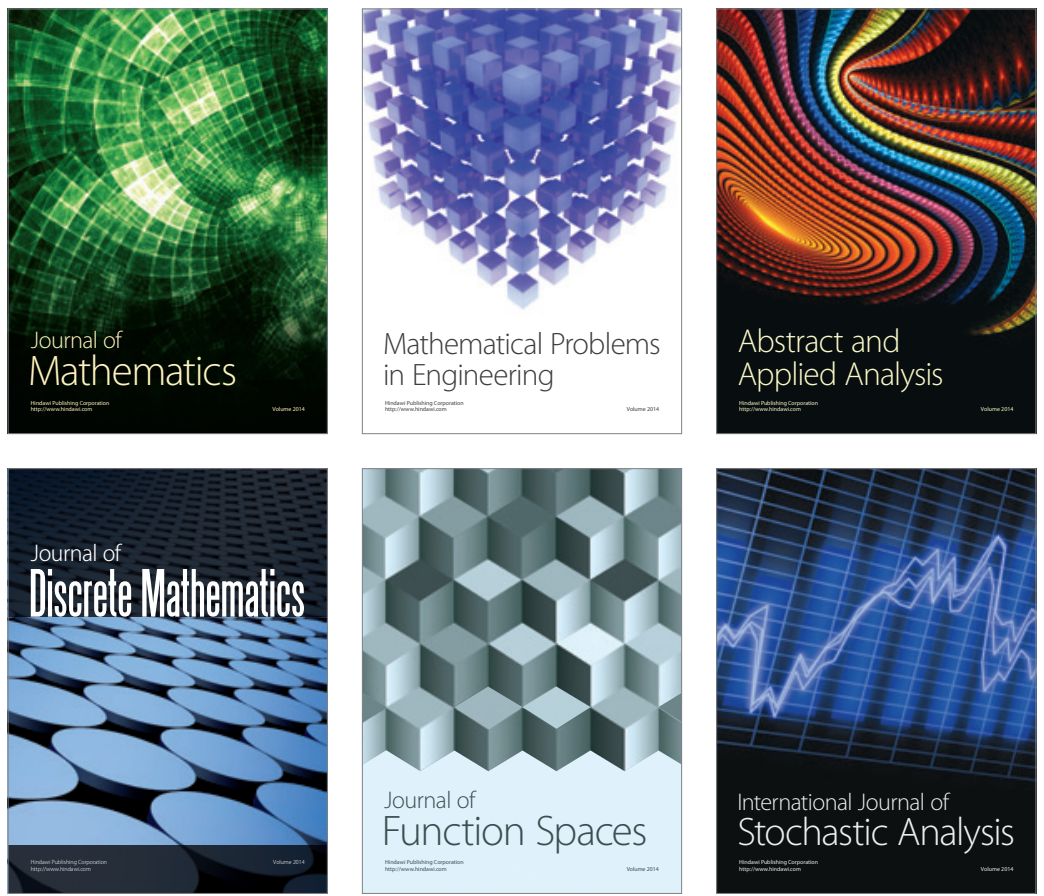

Journal of

Function Spaces

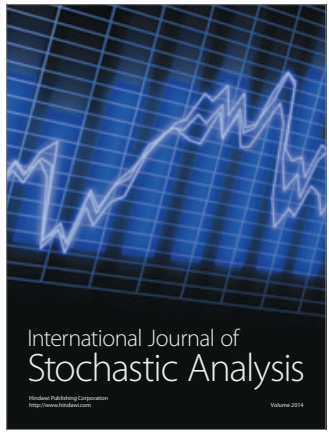

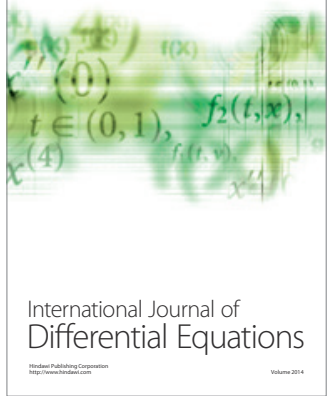
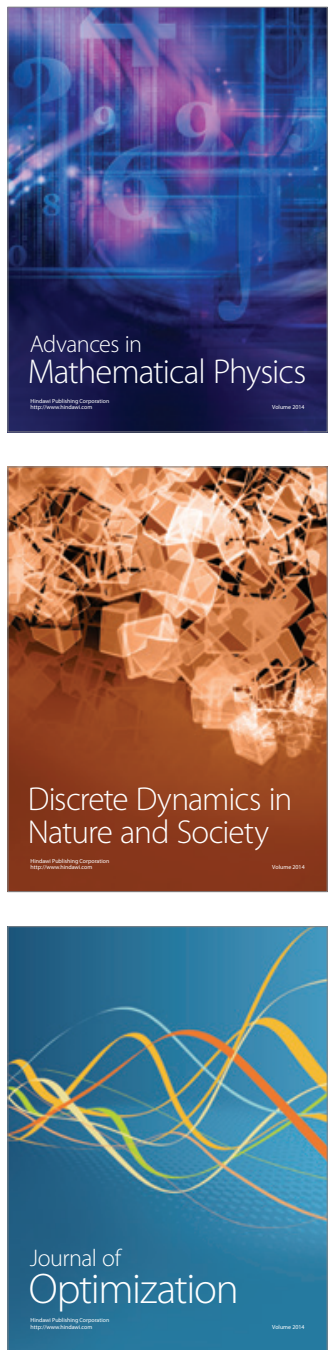